\title{
SUBJECTIVE WELLBEING AND PARTICIPATION OF 600 CHILDREN WITH CEREBRAL PALSY ACROSS EUROPE, VISITED AT 8-12 AND AGAIN AT 13-17 YEARS
}

\section{A. Colver}

Institute of Health and Society, Newcastle University, Newcastle upon Tyne, UK

The SPARCLE project explored the participation and quality of life of 800 children with cerebral palsy aged 8-12 years across nine European Regions. Then we visited the young people again aged 13-17 years.

Children with cerebral palsy are used as exemplars of disabled children because they may have impairments of movement, hearing and vision, intelligence and language; and there is a great range of severity.

SPARCLE is an epidemiological study with prespecified hypotheses, outcome measures and sampling procedures; all set out in its published protocol.

My talk will introduce the main concepts used which concern the social model of disability and the domains of the International Classification of Functioning, Disability and Health.

I will then present the main results concerning participation and quality of life and their determinants. These will include some of the preliminary findings from the longitudinal analyses. In particular I will draw out the results concerning the main hypothesis that "Children with similar severity of cerebral palsy will experience variable outcomes in different countries due to variation in environmental factors" 